\title{
Sekundär heilende Wunden
}

\section{Nachbegutachtung der multizentrischen Fallbeobachtungen mit naszierendem Sauerstoff}

Der naszierende (aktivierter, atomarer oder naszierender Sauerstoff genannt) Sauerstoff - $\mathrm{O}_{1}$ ist keine neue Entdeckung, sondern ist auch der Wirkstoff bei der medizinischen Ozontherapie, die schon seit mehr als 100 Jahren bei allen möglichen Krankheiten erfolgreich angewendet wird (Literaturen beim Verfasser). Dennoch darf diese Therapie nicht mit der Ozontherapie verwechselt oder gleichgesetzt werden.

\section{Zielsetzung der Studie}

Vom Juni 2009 bis 31.10.2010 wurden bei vier WundZentren in Österreich, der Schweiz und Deutschland die Frage erörtert, ob die klinischen Studienergebnisse aus dem Jahr 2008-2009 (www.wfi.ch/sites/dl/ download/ActiMaris-Clinicum_PUB_201003_CH.pdf/siehe auch PROCARE 0607/2011) bestätigt werden können oder ob es in der Zwischenzeit zu anderen oder gar negativen Erfahrungen kam.

Insbesondere interessierten dabei folgende Fragestellungen:

- Ist unter der Anwendung von ActiMaris ${ }^{\circ}$ Lösung oder Gel eine Reinigung der Wunde und Neubildung von Granulation im Sinne einer Förderung des Wundheilungsprozesses zu erkennen?

- Ist unter der Anwendung von ActiMaris $^{\bullet}$ Lösung (Nass-Trockenphase) eine Reduktion der Inflammationszeichen in der Wundumgebung zu erkennen?

- Ist unter der Anwendung von ActiMaris ${ }^{\circ}$ Lösung oder Gel eine Reduktion

1. Gerhard Kammerlander, MBA/DGKP / ZWM ${ }^{\circledast}$, Geschäftsführung, Akademie für zertifiziertes Wundmanagement ${ }^{\circledR}$ - KAMMERLANDER-WFI Embrach, Zürich, Schweiz und WKZ ${ }^{\circledR}$ - WundKompetenzZentrum, Linz

2 Petra Zweimüller, DGKS / AZWM ${ }^{\circledast}$, Pflegedienstleitung, $W K Z^{\oplus}$ - WundKompetenzZentrum, Linz

3 Anja Süss-Burghard, DGKS/ZWM ${ }^{\circledR}$, Leitung WundZentrum Krankenhaus Schwabing, München

4 Gertraud Pirkner, DGKS/ZWM ${ }^{\circledast}$, Lucie Schweiger DGKS/ZWM ${ }^{\circledast}$, Zentrum Chronische Wunden LKH Mariazell

5 Esther Locherer, DGKS / AZWM ${ }^{\circledR}$, Geschäftsführung $W^{\circledR}{ }^{\circledR}$-WundZentrum, Wundambulatorium Kallern-Hinterbühl, Schweiz oder Elimination von „übel riechenden" Gerüchen seitens der Wunde zu erkennen?

- Kann ActiMaris ${ }^{\bullet}$ vom klinischen Anwender einfach auf die Wunde appliziert werden?

- Kann ActiMaris ${ }^{\circledR}$ in tiefen Wunden mit zusätzlichen Wundfüllern wie Alginat, Hydrofaser etc. kombiniert appliziert werden?

- Wie gestaltet sich die Handhabung des Produktes im Rahmen des Verbandwechsels?

- Ist die Kompatibilität mit verschiedenen Produkten der Wundversorgung gegeben (klassische und moderne Verbandstoffe).

\section{Methodik}

In einer Behandlungsphase (Juni 2009-Oktober 2010) wurden insgesamt in allen WundZentren über 1.158 Patienten entsprechend den Anwendungshinweisen des Herstellers behandelt. Dabei wurde phasengerecht und wundtypgerecht gehandelt. Der professionelle Anwender entschied, wann die forte Version und wann die Standardversion verwendet wurde. Am häufigsten (mehr als $95 \%$ aller Anwendungen) kam die Wundspüllösung (Standardversion) zum Einsatz.

In dieser Bewertung wurden sekundär heilende Wunden unterschiedlicher Genese im Sinne einer phasengerechten Wundversorgung behandelt. Die Anwender waren durchweg erfahrene Wundbehandler entsprechend der Weiterbildung ZWM ${ }^{\circ}$ Zertifizierter Wundmanager. Sie wurden zu Beginn der Untersuchung in Handhabung und Produktindikation geschult. Sie waren angewiesen, eine phasengerechte Wundversorgung mit ActiMaris in Kombination mit den sonst üblichen Therapiemaßnahmen wie Kompressionstherapie, Druckentlastung etc. auszuführen.

Die Reinigung der Wunde sollte mit Hilfe der Nass-Trocken-Phase nach G. Kammerlander (www.wfi.ch/sites/dl/ download/Nass-Trocken_ZfW.pdf) erfolgen, die Hautpflege in der Wundumgebung phasen- und hauttypengerecht (www.wfi.ch/sites/dl/download/Haut-
pflege_Medizin.pdf) erfolgen.

Die Begutachtungen wurden in vier Behandlungszentren in Österreich, Deutschland und der Schweiz, ambulant und stationär im Zeitraum Mai 2009 bis Oktober 2010 durchgeführt. Die wissenschaftliche Leitung sowie das Monitoring oblag Gerhard Kammerlander, und Dr. med. Gerlinde Luch von der Akademie $\mathrm{ZWM}^{\circ}$ - KAMMERLANDER-WFI/WKZ WundKompetenzZentrum Linz.

\section{Ergebnisse}

Im Zeitraum Juni 2009 bis Oktober 2010 wurden insgesamt 1.158 Patienten mit ActiMaris ${ }^{\circ}$ mit behandelt (Nass-/Trockenphase, Benetzung von Wundprodukten wie Alginate, Hydrofaser,...). Dabei wurden mehr als 11.750 Einzelanwendungen durchgeführt.

\section{Zusammenfassung}

In der klinischen Anwendungsbeobachtung konnte ActiMaris ${ }^{\text {ent }}$ unter Alltagsbedingungen dokumentiert werden. Die Ergebnisse aus der ersten klinischen Anwendungsbeobachtung konnten vollumfänglich bestätigt werden. Es wurden keine zusätzlichen negativen Beobachtungen bzw. Erfahrungen gemacht.

\section{Interessenskonflikt}

Diese klinische Anwendungsbeobachtung wurde auf Antrag und Wunsch der Akademie für Zertifiziertes Wundmanagement initiiert und unentgeltlich durchgeführt. Die Produkte wurden in dieser zweiten Untersuchungsphase von den WundZentren bereits als Standardartikel geführt und selbst eingekauft.

Es besteht kein Interessenkonflikt im Sinne der Richtlinien des International Committee of Medical Journal Editors.

Korrespondenzadresse

Akademie für zertifiziertes Wundmanagement ${ }^{\circledR}$ - KAMMERLANDER-WFI Gerhard Kammerlander Taleggstrasse $23 \mathrm{CH}$ 8424 Embrach

Email: kammerlander@wfi.ch

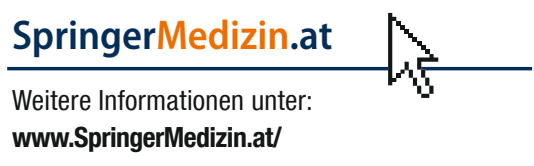

